\title{
Increased risk of Graves'ophthalmopathy in patients with increasing TRAb after radioiodine treatment and the impact of CTLA4 on TRAb titres
}

\author{
Bushra Shahida ${ }^{1} \cdot$ Kleoniki Tsoumani $^{2} \cdot$ Tereza Planck $^{2} \cdot$ Vijayachitra Modhukur $^{1} \cdot$ Pernilla Asp $^{3} \cdot$ Anna Sundlöv $^{4}$. \\ Jan Tennvall ${ }^{4} \cdot$ Peter Åsman ${ }^{5}$. Ola Lindgren ${ }^{2} \cdot$ Mikael Lantz $\mathbb{B}^{2}$
}

Received: 6 September 2021 / Accepted: 21 November 2021 / Published online: 2 December 2021

(c) The Author(s) 2021

\begin{abstract}
Introduction Treatment of Graves' disease (GD) with radioiodine increases the risk of developing Graves' ophthalmopathy (GO), and the link between thyroid and orbital tissue may be the presence of TSH-receptors. Radioiodine increases the titers of TRAb and the aim was to investigate the relationship between GO and TRAb titers after treatment with radioiodine and to define the impact of risk genes.

Methods GD patients without ophthalmopathy or previous treatment with radioiodine were prospectively included at treatment with radioiodine for hyperthyroidism. A follow-up was performed 1 year later for the registration of GO development. The study was performed at a University Hospital Clinic; a referral center of all patients treated with radioiodine in the south of Sweden. The main outcome measures were the development of TRAb, anti-TPO, and anti-TG after 3 months and GO after 12 months and relationship to the genetic background (HLA, CTLA-4, and CYR61).

Results Three months of radioiodine TRAb titers increased in two thirds of patients $(p<0.0005)$ but not in the other third. Anti-TPO titers were associated with TRAb $(R=0.362, p<0.0001)$ but not anti-TG. At follow-up 1 year later $(n=204) 32$ patients developed GO with a proportion of $70 \%$ in the group increasing in TRAb titers and $30 \%$ in the group with unchanged or lower TRAb titers ( $p$-value $<0.0005)$. Patients with GO had higher titers of TRAb than patients without GO. CTLA-4 (rs231775 SNP) was significantly $(p<0.005)$ associated with TRAb titers above the median three months after radioiodine.

Conclusions The increase in TRAb titers after treatment with radioiodine is associated with GO and a genetic variation in CTLA-4 is associated with higher titers of TRAb.
\end{abstract}

Keywords Graves' disease $\cdot$ TRAb $\cdot$ Anti-TPO $\cdot$ Anti-TG $\cdot$ Radioiodine $\cdot$ CTLA-4

Mikael Lantz

Mikael.Lantz@med.lu.se

1 Department of Clinical Sciences Malmö, Diabetes and Endocrinology, Lund University, Malmö, Sweden

2 Department of Endocrinology, Department of Clinical Sciences, Skåne University Hospital, Lund University, Malmö and Lund, Sweden

3 Department of Oncology, Skåne University Hospital, Lund, Sweden

4 Department of Oncology, Department of Clinical Sciences, Skåne University Hospital, Lund University, Lund, Sweden

5 Department of Clinical Sciences Malmö, Ophthalmology, Skåne University Hospital, Department of Ophthalmology, Lund University, Malmö, Sweden

\section{Introduction}

In Graves' disease (GD), immunocompetent cells infiltrate thyroid tissue with the release of TSH-receptor stimulating antibodies (TRAb), resulting in hyperthyroidism. The triggering of the autoimmune response depends on the interplay of genetic [1,2] and environmental factors [3]. A strong risk factor is tobacco smoking which results in higher TRAb at diagnosis of GD and during treatment with thiamazole than non-smokers [3, 4].

Smoking has been shown to increase TRAb titers and the risk of development of GO both at and after diagnosis of GD $[5,6]$. Treatment of GD with radioiodine is a risk factor for the development of $\mathrm{GO}$ and might be mediated by activating TRAb [7]. Laurberg et al. has shown that 
treatment with radioiodine increases TRAb with a maximum after 3 months which is in contrast to treatment with anti-thyroid drugs (ATD) or thyroidectomy, where TRAb slowly declines without a prior increase [8]. It has been suggested that the increase in TRAb after radioiodine treatment is mediated by a transient release of thyroid antigens $[9,10]$. However this assumption has been difficult to confirm. In some patients the increase in TRAb persists for several years, indicating the existence of other mechanisms involving the activation of specific immunocompetent cells or a prolonged effect of radioiodine on immunocompetent cells. Irradiated Hashimoto lymphocytes have been studied in vitro, and it was proposed that irradiated lymphocytes of the thyroid are important insynthesising of autoantibodies in response to 131-Iodine [11].

We have previously identified immediate early genes (IEGs) overexpressed in orbital tissue from patients with severe GO [12]. These genes have later been investigated for the presence of gene polymorphisms and we found an association of CYR61 with GD and GO, and an increased risk for GO in smokers (OR 4.75) [13]. Later, in a microarray analysis of human orbital tissue, we showed that the IEGs exhibited higher expression in smokers than nonsmokers [14]. Cytotoxic T-lymphocyte antigen (CTLA-4) and human leukocyte antigen (HLA) are other genes with known association to GD [2]. There are no known genes associated only with GO.

In a previous study on the treatment of GD patients with radioiodine we have defined one group with increases in TRAb, thyroid peroxidase antibodies (anti-TPO), thyroglobulin antibodies (anti-TG), and another group with no increase of these thyroid antibodies after treatment with radioiodine [15]. We have now increased the number of patients and followed these patients for at least 1 year.

The present study aimed to investigate the relationship between TRAb and GO development and define the impact of risk genes.

\section{Material and methods}

\section{Subjects and treatment}

Treatments with 131-iodine in Skåne County are centralized to the department of Oncology in Lund where all patients with hyperthyroidism are referred if the patient's ordinary clinician decides that radioiodine is the best treatment choce.

This prospective observational study included GD patients, without GO, admitted to the Department of Oncology for treatment with radioiodine from August 2016 until August $2018(n=204)$ and followed up 1 year later for the development of GO
TRAb, anti-TPO, and anti-TG were measured before and 3 months after 131-iodine treatment and the fold changes were calculated.

Patients without $\mathrm{GO}$ at radioiodine treatment but with risk factors for the development of GO were prescribed (by the treating clinician), prophylaxis with prednisolone. These patients $(n=45)$ received prednisolone $30 \mathrm{mg}$ per day, independently of body weight, for 1 month and after that the dose was slowly decreased during the next 2 months and stopped after 3 months.

The patients were classified as having GD based on clinical signs, increased thyroid hormones and suppressed $\mathrm{TSH}$, the presence of TRAb, and/or a diffuse uptake on thyroid technetium scintigraphy; all patients performed this investigation. One year, later all patients were screened for the GO development (or not) by an endocrinologist or an ophthalmologist at their home clinic in Skåne. All patients received a questionnaire and DNA swab for self-collection of DNA from the buccal mucosa which 130 out of 204 patients accepted and sent in an envelope to our research laboratory. DNA was amplified and analysed for the presence of polymorphisms in known risk genes for GD and GO; cytotoxic T-lymphocyte antigen 4 (CTLA4), human leukocyte antigen-DRB (HLA-DRB) and, cysteine-rich 61 (CYR61).

In total 204 patients not previously treated with radioiodine were included. The following parameters were registered; age, sex, born in Sweden, tobacco smoking, duration of GD and GO, and treatment with corticosteroids (Table 1).

The primary endpoint was development of GO and the number of patients planned to be included was approximately 200 patients to obtain approximately 40

Table 1 Changes in TRAb after treatment with radioiodine and relation to clinical parameteters

\begin{tabular}{llll}
\hline Fold change TRAb & $<1.1$ & $\geq 1.1$ & $P$ value \\
\hline Patients (\%) & $57(31)$ & $125(69)$ & \\
Females (\%) & $45(79)$ & $97(78)$ & 0.84 \\
Age males and females, years & $56 \pm 17$ & $54 \pm 16$ & 0.67 \\
Age females, years & $54 \pm 17$ & $52 \pm 16$ & 0.49 \\
Smokers (\%) & $8(14)$ & $31(25)$ & 0.10 \\
Born outside Sweden (\%) & $12(21)$ & $35(28)$ & 0.32 \\
Treatment with Corticosteroids (\%) & $41(73)$ & $79(63)$ & 0.23 \\
Treatment with >120 Gy & $10(18)$ & $21(16)$ & 0.99 \\
Duration of GD, months & $12(5-25)$ & $8(3-33)$ & 0.48 \\
Primary treatment with ATD (\%) & $37(65)$ & $70(56)$ & 0.33 \\
Duration of GO, months & $6 \pm 6$ & $5 \pm 3$ & 0.52 \\
Treatment with Corticosteroids in & $2(22)$ & $4(17)$ & 0.99 \\
GO patients & & & \\
GO smokers & 0 & 2 & 0.99 \\
\hline
\end{tabular}


ophthalmopathy patients, which is based on the knowledge of a previous Swedish multicenter study [7] where $20 \%$ developed ophthalmopathy during the first year. Based on our previous study on radioiodine where two thirds increased in TRAb and one third were unchanged we assumed that GO was distributed similarly, which should give a significant difference of ophthalmopathy between groups. The secondary endpoint was the presence of polymorphisms in known risk genes in patients with high TRAb.

As a clinical routine 120 Gray was used, but in some patients up to 300 Gray was administered if the aim was to decrease the risk of relapse. Methimazole or propylthiouracil with or without L-thyroxine was used in some patients before radioiodine and was stopped 2 weeks (1 week before the start of iodine uptake measurement) before administering of 131-iodine. The ALARA (as low as reasonably achievable) principle was used when defining the activity needed to achieve the described doses and the activity (MBq) was estimated by use of the following formula: Dose (D) $\times$ Mass $(\mathrm{m}) / 0.043 \times$ uptake day zero $(\mathrm{U} 0) \times$ effective half-life (Teff). U0 and Teff were calculated from the iodine uptake at $24 \mathrm{~h}$ and 7 days. The thyroid mass was calculated from $99 \mathrm{mTc}$-pertechneate scintigraphy.

The proportion of absorbed doses used were distributed as follows: $120 \mathrm{~Gy}(78 \%),>120-300 \mathrm{~Gy}(22 \%)$.

\section{Gene polymorphisms analysis and DNA extraction}

In total five single nucleotide polymorphisms (SNPs) were genotyped in 127 patients (Table 2); Two SNPs, rs1378228 and rs12656618, in CYR61 and two in CTLA4, rs3087243,

Table 2 Characteristics of genotyped patients

\begin{tabular}{lll}
\hline & $\begin{array}{l}\text { TRAb (IU/L) }< \\
\text { median }(15 \text { IU/L) }\end{array}$ & $\begin{array}{l}\text { TRAb (IU/L) } \geq \\
\text { median }(15 \text { IU/L) }\end{array}$ \\
\hline$N$ & 57 & 60 \\
Age & $55 \pm 18$ & $54 \pm 15$ \\
Sex & & $15(25)$ \\
Male & $13(23)$ & $45(75)$ \\
Female & $44(77)$ & \\
Ethnicity & & $47(78)$ \\
Born in Sweden & $44(77)$ & $7(12)$ \\
Born in Europe & $7(12)$ & $6(10)$ \\
Born outside Europe & $6(11)$ & $10(17)$ \\
Smokers & $12(21)$ & $39(65)$ \\
Non-smoker & $41(72)$ & $11(18)$ \\
Missing & $4(7)$ & $31(24-39)$ \\
$*$ TRAb IU/L (median & $6(3-9)$ & \\
15 IU/L) & & \\
\hline
\end{tabular}

Missing information on ten patients

Values expressed are means $( \pm \mathrm{SD})$ and presented as $n(\%)$ unless otherwise stated and rs231775, were chosen for analysis based on previous studies on GD and GO (13) Also, a tag SNP, rs6457617, HLA Class II, DR Beta 1 (HLA-DR-DQQ was analysed as previously described [16].

DNA was collected for genotyping using buccal swabs, then extracted using QiAamp UCP DNA Micro Kit (Qiagen, Sweden) and amplified using Repli-g Screening Kit (Qiagen, Sweden). SNPs were genotyped by TaqMan Allelic Discrimination Assay using the Quantstudio 7 Flex system (Applied Biosystems by Life Technologies, Sweden).

The minor allele frequency (MAF) for all SNPs was $>$ 0.05. One SNP (rs12756618 in CYR61) failed the Hardy-Weinberg equilibrium and was excluded from the analysis.

The standard statistical analysis approach was used to find the association of TRAb $<$ median/TRAb $>$ median and GO/no GO association. A linear regression model was used with smoking and gender as covariates. The data are presented as odds ratios (ORs) with $95 \%$ confidence intervals (CIs). The $p$-values are based on additive models for the genetic variants. All genetic analyses were performed using PLINK version 1.0 (http://pngu.mgh.harvard.edu/ purcell/ plink/index.shtml).

\section{Assays for antibodies}

TRAb was measured with a competitive Electro Chem Luminiscens Immunoassay (ECLI) according to the manufactures instructions (Roche). The limit of detection was 0.3 IU/L, CV 5\% at 16 IU/L. The cut-off for a positive value of TRAb was $>1 \mathrm{kIU} / \mathrm{L}$.

Anti-TPO titer was measured with a competitive sandwich ELISA (Roche) according to the manufactures instructions (detection limit $5 \mathrm{kIU} / \mathrm{L}, \mathrm{CV} 11 \%$ at $34 \mathrm{kIU} / \mathrm{L}$. The cut-off for a positive value of anti-TPO was $>34 \mathrm{kIU} / \mathrm{L}$.

Anti-TG was measured with a competitive sandwich ELISA (Roche) according to the manufactures instructions (detection limit $10 \mathrm{kIU} / \mathrm{L}, \mathrm{CV} 10 \%$ at $73 \mathrm{kIU} / \mathrm{L}$ ). The cutoff for a positive value of anti-TG was $>115 \mathrm{kIU} / \mathrm{L}$.

Samples were analysed in routine clinical laboratory at the Department of Clinical Chemistry in Malmö and Lund.

\section{Statistics}

The fold changes of thyroid antibodies were calculated. A change of 1.1 or more was judged as an increase and if lower than 1.1 the change was judged as unchanged or decreased. The $t$-test (continuous variables), chi-square test (categorical variables), and bi-nomial test were used to assess the statistical significance of differences between the groups. Linear regression analysis was used to study the correlations between the parameters fold change of TRAb, anti, TPO, and anti-TG. All statistical analyses were carried 
A

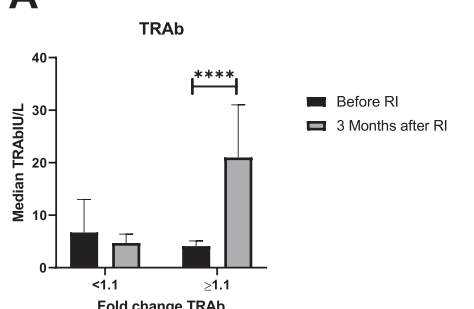

D

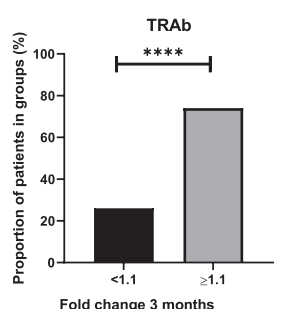

B

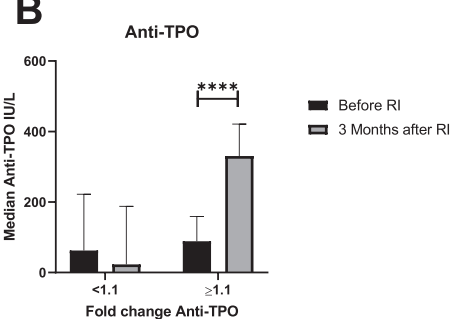

E

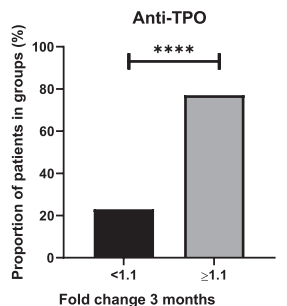

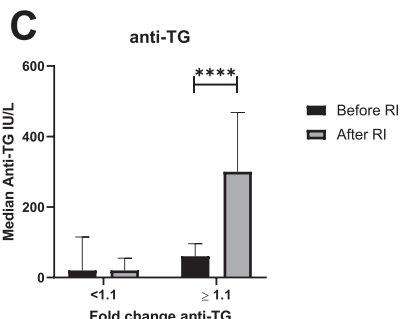

$\mathbf{F}$

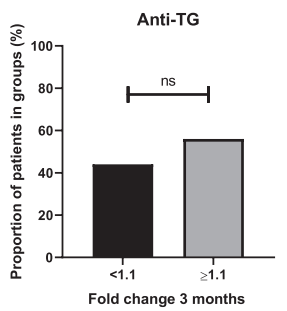

Fig. 1 Fold change and proportion of TRAb (A, D), anti-TPO (B, E), and anti-TG (C, F) 3 months after treatment of Graves' disease with radioiodine. The median values of TRAb, anti-TPO and anti TG before and after radioiodine in the group with fold change $<1.1$ and in the group with fold change $>/=1.1$ were all significant with $p$-values $<$ 0.0001 ( $t$-test). Differences in proportion was calculated with a binomial test and differences in median values with a $t$-test out using the SPSS 22.0 statistical software (SPSS, Chicago, IL, USA) or Graph Pad prism 8.0 The significance levels were $* p<0.05 ;{ }^{* *} p<0.01 ; * * * p<0.001, * * * * p<$ 0.0001 .

\section{Results}

At the start of treatment with radioiodine 204 patients were registered and thyroid antibodies; TRAb, anti-TPO, anti TG were analysed before treatment with radioiodine, and 3 months after radioiodine the antibody analysis was repeated for detection of patients that showed an increase of 1.1 or more. The cut-off was set based on the knowledge of the variation coefficient for the antibody assays.

We found two groups according to antibody response 3 months after radioiodine; one group increased in titers of TRAb anti-TPO and anti-TG (70\% of patients). Another group showed a decrease in titers of antibodies or were unchanged (30\% of patients) (Fig. 1). There was not only a significant increase or decrease in fold change of antibody titers 3 months after radioiodine, but also a significant increase or decrease in median values of all three antibodies (Fig. 1). Three months after treatment, a correlation with radioiodine was found for titers of TRAb and anti-TPO (Fig. 2) but not for anti-TG (data not shown).

All 204 patients were followed for at least 1 year after treatment with radioiodine to identify the development of GO and correlate GO to the antibody response. We found an overrepresentation of GO in the group with increased titers of TRAb and anti-TPO with a proportion of GO

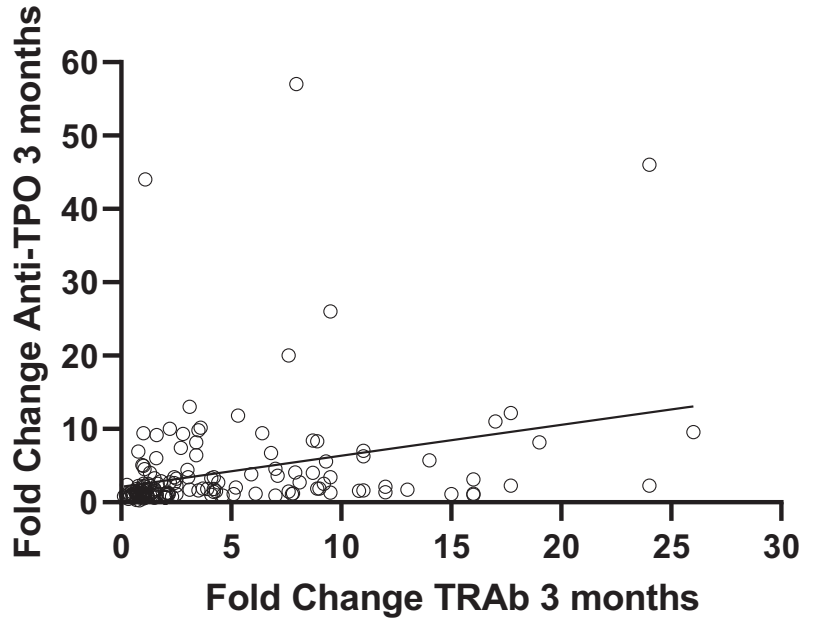

Fig. 2 Correlation of fold change in TRAb and anti-TPO 3 months after treatment with $\mathrm{RI}(R=0.362, p<0.0001)$

patients at follow-up after 1 year of $70 \%$ versus $30 \%$ in the group with no increase in antibody titers three months after radioiodine (Fig. 3A). If we excluded those patients with GO and low or unchanged titers of TRAb or anti-TPO (Fig. 3B) who had received treatment with prednisolone the effect of antibodies was more pronounced with a proportion of GO of $80 \%$ versus $20 \%$ for TRAb and similar proportions for anti-TPO (Fig. 3B). When analysing GO patients for TRAb and anti-TPO the median values were significantly different, with higher values in the group of GO patients with increased titers of TRAb compared with unchanged or lower values (Fig. 3C). If dividing the material in patients with or without GO the median TRAb 
Fig. 3 Development of Graves' ophthalmopathy 1 year after RI in the whole group. A Increased TRAb $(n=32)$ or anti-TPO $(n=23)$ compared with no increase in TRAb or anti-TPO, 3 months after RI. Proportion of all GO patients in TRAb $(n=32)$ or anti-TPO $(n=23)$ including prednisolone treated patients in the group with no increase in antibodies.

B Proportion of patients where prednisolone treated patients were excluded in the group with no increase in TRAb $(n=26)$ or anti-TPO $(n=20)$. C GO patients with median values of TRAb $(n=26)$ and anti-TPO $(n=20)$ in patients 3 months after radioiodine treatment. D Median values of TRAb in patients with and without GO with a dotted line that shows the median value of TRAb in all patients. Differences in proportion was calculated with a binomial test and differences in median values the $t$-test was used. Significance is shown in figures

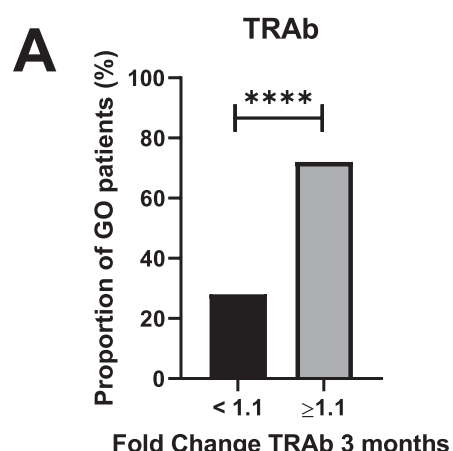

Fold Change TRAb 3 months

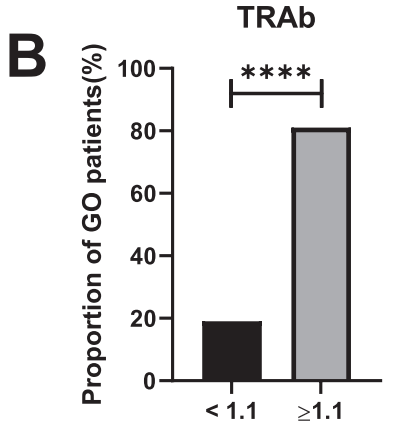

Fold Change TRAb 3 months

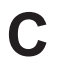

TRAb

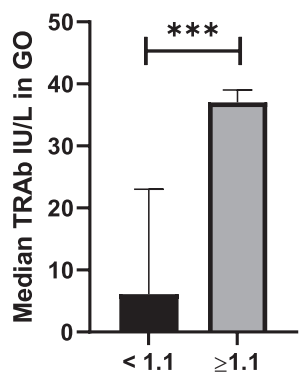

Fold Change TRAb 3 months
Anti-TPO

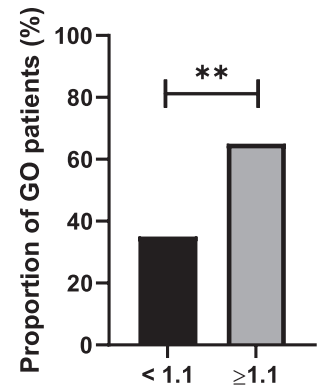

Fold Change Anti-TPO 3 months

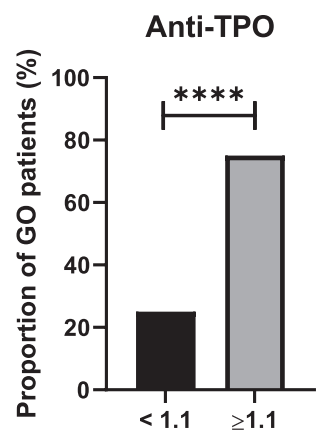

Fold Change Anti-TPO 3 months

Anti-TPO

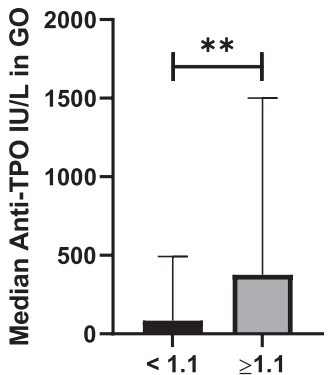

Fold Change Anti-TPO 3 months

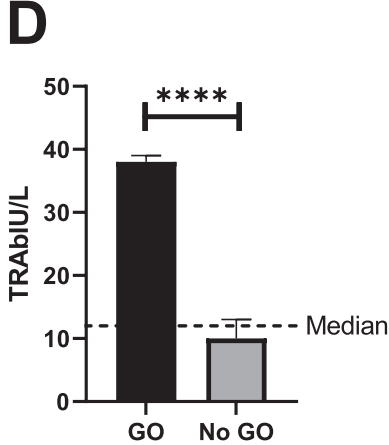

titers were significantly higher in patients with GO and the majority of patients with GO have antibody titers above the median of the entire material (Fig. 3D).
We also tried to define an association of increase or decrease in antibody titers and clinical parameters previously associated with the incidence of GD, e.g., sex, 
Table 3 SNPs in GD patients treated with radioiodine.

\begin{tabular}{|c|c|c|c|c|c|c|}
\hline Gene & SNP & $\begin{array}{l}\text { Allele frequency } \\
\text { TRAb (IU/L) } \\
<\text { median } \\
(15 \text { IU/L) }\end{array}$ & $\begin{array}{l}\text { Allele } \\
\text { Frequency } \\
\text { TRAb (IU/L) } \\
\geq \text { median } \\
(15 \mathrm{IU} / \mathrm{L})\end{array}$ & $\begin{array}{l}\text { Associated } \\
\text { Allele }\end{array}$ & $\begin{array}{l}\text { OR for associated } \\
\text { allele }(95 \% \mathrm{CI})\end{array}$ & $p$-value \\
\hline \multicolumn{7}{|c|}{ A. Association with TRAb below and above median $15 \mathrm{IU} / \mathrm{L}$} \\
\hline CYR61 & rs1378228 & 0.35 & 0.39 & $\mathrm{~T}$ & $1.19(0.72-1.99)$ & 0.500 \\
\hline \multirow[t]{2}{*}{ CTLA4 } & rs3087243 & 0.37 & 0.42 & A & $1.28(0.77-2.12)$ & 0.334 \\
\hline & rs 231775 & 0.46 & 0.29 & G & $0.48(0.28-0.80)$ & 0.005 \\
\hline HLA-DRB 1 & rs6457617 & 0.42 & 0.42 & $\mathrm{C}$ & $1.30(0.794-2.14)$ & 0.295 \\
\hline \multicolumn{7}{|c|}{ B. Association with $G O$} \\
\hline Gene & SNP & $\begin{array}{l}\text { Allele frequency } \\
\text { in patients } \\
\text { without GO }\end{array}$ & $\begin{array}{l}\text { Allele } \\
\text { frequency in } \\
\text { patients } \\
\text { with GO }\end{array}$ & $\begin{array}{l}\text { Associated } \\
\text { allele }\end{array}$ & $\begin{array}{l}\text { OR for associated } \\
\text { allele }(95 \% \mathrm{CI})\end{array}$ & $p$-value \\
\hline CYR61 & rs1378228 & 0.36 & 0.4 & $\mathrm{~T}$ & 1.181 & 0.58 \\
\hline \multirow[t]{2}{*}{ CTLA4 } & rs3087243 & 0.37 & 0.48 & A & 1.62 & 0.10 \\
\hline & rs 231775 & 0.41 & 0.28 & G & 0.56 & 0.07 \\
\hline HLA-DRB1 & rs6457617 & 0.53 & 0.53 & $\mathrm{C}$ & 1.56 & 0.13 \\
\hline
\end{tabular}

ethnic background, smoking, and age, but we found no correlation with these parameters (Table 1). We also studied factors that might affect treatment with radioiodine (treatment dose, duration of disease, primary treatment) and found no factor that affected the antibody response (Table 1). Lastly we also studied factors that might increase the presence and risk of GO (smoking, duration of GO, treatment with steroids), these were not different between the antibody response groups (Table 1). The size of the thyroid was determined by technetium scintigraphy but did not affect the two defined groups (data not shown).

\section{Genotyping of Graves' patients treated with radioiodine}

We analysed SNPs in HLA, CTLA-4, and CYR61 in patients $(n=127)$ treated with radioiodine (Table 2$)$ to define the genetic background in the development of GO and antibody response of TRAb, anti-TPO, and anti-TG. The response was divided into patients with increased or decreased antibody titers 3 months after radioiodine.

There was no significant difference in the presence of described SNPs between these groups nor did we detect a significant difference between patients with or without GO, although the allele frequency was lower in patients with GO (OR 0.56, $p=0.07$ ) (Table 3). However, when analysing the material by dividing TRAb titers into groups above and below the median (15 IU/L) after treatment with radioiodine, the allele frequency of the rs231775 SNP in CTLA4 was significantly changed between groups (OR $0.48, p=$ 0.005 ) and associated with TRAb titers above the median which was confirmed by linear regression analysis of TRAb (data not shown), which was in contrast to anti-TPO and anti-TG which showed no significant association (Table 3).

\section{Discussion}

Strong risk factors for the development of GO are treatment with radioiodine and smoking, but mechanisms for these effects are, in most cases, not defined. The link between changes in thyroid tissue after radioiodine and orbital tissues is unknown.

We have shown that 3 months after treatment with radioiodine, two groups of patients were identified according to antibody response, one group that did not increase in titers of TRAb, anti-TPO, and anti-TG (approximately 30\% of the patients) and one group that increased in antibody titers (approximately $70 \%$ of the patients). This has previously been demonstrated in a smaller group of GD patients [15].

The second observation was that the risk of developing GO we elevated in patients which increased in TRAb titers 3 months after radioiodine.

The third observation was that patients with a polymorphism in CTLA4 developed higher titers of TRAb than in patients without this polymorphism.

Laurberg et al. have previously shown that radioiodine treatment increases TRAb titers already 1 month after treatment and with a maximal response after 3 months [8]. We have identified two subgroups, one subgroup does not increase in antibody titers and the other group increased in thyroid antibody titers after treatment with radioiodine. The latter group might be important to identify because 
prednisolone is most often given to all risk patients at the start of radioiodine to prevent GO. Here we show that GO preferentially develops in the group that increases in thyroid antibody titers after 3 months indicating that this group should receive pre-treatment with prednisolone. In our study, we have treated risk patients with prednisolone at the start of radioiodine with a decrease of prednisolone 1 month later and with termination after 3 months when antibody titers are at maximal levels, and the prednisolone dose is low. Further studies are needed to define the kinetics of the increase in TRAb titers resulting in new routines for pretreatment with prednisolone.

We and others have previously shown that treatment with radioiodine increases the risk of developing GO, but the mechanism has not been defined [7, 17, 18]. Here we show that the link between, the thyroid and the orbita tissue might be a release of TRAb and anti-TPO presumably from infiltrating immunocompetent cells of the thyroid, which later affect the orbital tissue in risk patients. One possible mechanism is that radioiodine decreases all immunocompetent cells, but the antibody producing cells have a higher proliferation rate than immunosuppressive cells, resulting in an imbalance between the described cells with an uncontrolled release of thyroid antibodies. In vitro it has been shown that a mixture of immunocompetent cells in Hashimoto produces thyroid specific antibodies after irradiation, and this effect was dose dependent [11]. We have previously studied the effect of the dose of radioiodine, but we could not confirm this in vivo [15].

Another hypothesis is that a transient increase in thyroid antigen like thyroglobulin will be released when irradiation destroys cells [9, 10]. However, we found no association between TRAb and thyroglobulin titers compared with TRAb and anti-TPO titers after treatment with radioiodine. Also in some cases thyroid antibodies might persist for several years after radioiodine indicating that other mechanisms exist.

Active smoking is another strong risk factor for autoimmune thyroiditis (AIT), GD and GO [3]. We and others have shown that TRAb persists at higher titers than nonsmokers during treatment with methimazole [4]. In orbital tissues we have studied other risk factors for $\mathrm{GO}$ and found that smokers have increased expression of CYR61 [14]. Smoking is a risk factor for AIT and for other autoimmune diseases like rheumatoid arthritis, these patients show higher activity parameters in smokers [19].

It is well established that HLA and CTLA-4 are associated with GD, but there is lack of a specific genotype to GO. However, in a previous microarray we have shown that a matrix protein, CYR61, is increased in patients with severe GO in the active phase with a decrease in the chronic phase [12]. In another microarray of orbital tissue, we have also shown that CYR61 is preferentially increased in smokers compared with non-smokers [14]. In a genotyping study, we have demonstrated an increased risk of developing GO in smokersassociated with CYR61 [13].

In the present study, we have found a new association of TRAb titers elevated above the median and rs231775 in CTLA-4. The variant is located in exon $1+49$ and it was shown that the $A$ to $G$ substitution at this location is linked with GD [20]. A study by Ban et al. found that the genotype $\mathrm{GG}$ in the CTLA4 $\mathrm{A} / \mathrm{G}_{49} \mathrm{SNP}$ resulted in a reduced increase in $\mathrm{T}$-cell proliferation compared with the AA genotype [21].

Furthermore a previous study showed that a gene polymorphism in CTLA-4 contributes to the pathogenesis of GD because when peripheral blood cells were incubated with a monoclonal anti-human CTLA-4 monoclonal antibody resulted in increased blood cell proliferation [22]. It has also been demonstrated that a splice variant of CTLA-4 results in the release of a soluble form of CTLA4 with increased levels in serum from patients with GD and that this soluble form bind to CD80/CD86, resulting in inhibition of T-cell activation [23]. Later, it was shown that increased levels of soluble CTLA-4 correlates with the severity of $\mathrm{GO}$ and that a genetic variation in the CTLA-4 gene region partially determines the level of its soluble form [24]. Other autoimmune conditions, like myasthenia gravis, SLE, coeliac disease and type 1 diabetes, are associated with soluble forms of CTLA-4, which has been reviewed by Saverino et al. [25]. However, no study describes an association between a SNP in CTLA-4 and increased autoantibody titers as in our study with increased titers of TRAb.

To conclude, we have demonstrated that the increase in TRAb titers after treatment with radioiodine of GD patients without GO is associated with later development of GO compared to a group that does not increase in TRAb titers. Also we have demonstrated that a genetic variation in CTLA-4 is associated with higher titers of TRAb after treatment with radioiodine.

Acknowledgements We are grateful to the nurses at the department of Oncology for the help with the blood tests and Perparim Cerri for amplifying DNA.

Author contributions The authors Mikael Lantz and Ola Lindgren treated these patients and collected data and DNA. Bushra Shahida and Vijayachitra Modhukur genotyped all patients and performed bioinformatic analysis. All authors have made a substantial contribution to the design of the study, acquisition and interpretation of data, as well as revising it critically for important intellectual content. 
Funding This work was supported by grants from the Faculty of Medicine at Lund University, the Påhlsson Foundation, the Skåne Research Foundation and the Research Funds of Skåne University Hospital.

Funding Open access funding provided by Lund University.

\section{Compliance with ethical standards}

Conflict of interest The authors declare no competing interests.

Ethical approval The regional research ethics committee approved the study in Lund, Sweden (project number 160610). Informed consent was obtained from participating patients after a full explanation of the purpose and nature of all procedures used.

Consent to participate The patients provided written informed consent for disclosing clinical and imaging data.

Publisher's note Springer Nature remains neutral with regard to jurisdictional claims in published maps and institutional affiliations.

Open Access This article is licensed under a Creative Commons Attribution 4.0 International License, which permits use, sharing, adaptation, distribution and reproduction in any medium or format, as long as you give appropriate credit to the original author(s) and the source, provide a link to the Creative Commons license, and indicate if changes were made. The images or other third party material in this article are included in the article's Creative Commons license, unless indicated otherwise in a credit line to the material. If material is not included in the article's Creative Commons license and your intended use is not permitted by statutory regulation or exceeds the permitted use, you will need to obtain permission directly from the copyright holder. To view a copy of this license, visit http://creativecommons. org/licenses/by/4.0/.

\section{References}

1. Y. Tomer, T.F. Davies, Searching for the autoimmune thyroid disease susceptibility genes: from gene mapping to gene function. Endocr. Rev. 24(5), 694-717 (2003).

2. T.F. Davies, Really significant genes for autoimmune thyroid disease do not exist - so how can we predict disease? Thyroid $\mathbf{1 7}$ (11), 1027-1029 (2007).

3. W.M. Wiersinga, Smoking and thyroid. Clin. Endocrinol. 79(2), 145-151 (2013).

4. M. Lantz, J. Calissendorff, F. Träisk, L. Tallstedt, T. Planck, O. Törring, B. Hallengren, P. Åsman, Adjuvant treatment of Graves' disease with diclofenac-safety, effects on ophthalmopathy and antibody concentrations. Eur. Thyroid J. 5(1), 50-57 (2016).

5. D.H.C. Khoo, S.C. Ho, L.L. Seah, K.S. Fong, E.S. Tai, S.P. Chee, P.H.K. Eng, S.E. Aw, A.C.K. Fok, The combination of absent thyroid peroxidise antibodies and high thyroid-stimulating immunoglobulin levels in GD identifies a group at markedly increased risk of ophthalmopathy. Thyroid 9(12), 1175-1180 (1999).

6. M. Lantz, T. Planck, P. Åsman, B. Hallengren, Increased TRAb and/or low anti-TPO titers at diagnosis of Graves' disease (GD) are associated with an increased risk of developing ophthalmopathy after onset of GD. Exp. Clin. Endocrinol. Diabetes 122(2), 113-118 (2014).
7. F. Träisk, M. Abraham-Nordling, G. Berg, J. Calissendorff, B. Hallengren, P. Hedner, M. Lantz, E. Nyström, L. Tallstedt, A. Taube, O. Törring, G. Wallin, P. Åsman, G. Lundell, Thyroid associated ophthalmopathy ocurrence after treatment for Graves' hyperthyroidism with iodine-131 or anti-thyroid drugs. J. Clin. Endocrinol. Metab. 94(10), 3700-3707 (2009).

8. P. Laurberg, G. Wallin, L. Tallstedt, M. Abraham-Nordling, G. Lundell, O. Törring, TSH-receptor autoimmunity in Graves's disease after therapy with anti-thyroid drugs, surgery, or radioiodine: a 5 year prospective randomized study. Eur. J. Endocrinol. 158(1), 69-75 (2008).

9. S. Atkinson, A.M. McGregor, P. Kendall-Taylor, M.M. Peterson, B.R. Smith, Effect of radioiodine on stimulatory activity of Graves' immunoglobulins. Clin. Endocrinol. 16(6), 537-543 (1982).

10. C.S. Teng, R.T. Yeung, R.K. Khoo, T.T. Alagaratnam, A prospective study of the changes in thyrotropin binding inhibitory immunoglobulins in Graves' disease treated by subtotal thyroidectomy or radioactive iodine. J. Clin. Endocrinol. Metab. 50 (6), 1005-1010 (1980).

11. A.M. McGregor, B. Rees Smith, S. McLachlan, R. Hall, Effect of irradiation on thyroid- antibody production. Lancet 2(8140), 442-444 (1979).

12. M. Lantz, T. Vondrichova, H. Parikh, C. Frenander, M. Ridderstråle, P. Åsman P, M. Åberg, L. Groop, B. Hallengren, Overexpression of immediate early genes in active Graves' ophthalmopathy. J. Clin. Endocrinol. Metab. 90(8), 4784-4791 (2005).

13. T. Planck, B. Shahida, M. Sjögren, L. Groop, B. Hallengren, M. Lantz, Association of polymorphisms in CTLA4, BTG2, CYR61, ZFP36 and SCD with Graves' disease and ophthalmopathy. Thyroid 24(7), 1156-1161 (2014).

14. T. Planck, B. Shahida, H. Parikh, K. Ström, P. Åsman, H. Brorson, B. Hallengren, M. Lantz, Immediate early genes are overexpressed in smokers with Graves'ophthalmopathy compared to non-smokers. Thyroid 24(10), 1524-1532 (2014).

15. O. Lindgren, P. Asp, A. Sundlöv, J. Tennvall, B. Shahida, T. Planck, P. Åsman, M. Lantz, The effect of radioiodine treatment on TRAb, anti-TPO and anti-TG in Graves' disease. Eur. Thyroid J. 8(2), 64-69 (2019).

16. M.J. Simmonds, S.C.L. Gough, Unravelling the genetic complexity of autoimmune thyroid disease: HLA, CTLA-4 and beyond. Clin. Exp. Immunol. 136(1), 1-10 (2004).

17. L. Bartalena, C. Marcocci, F. Bogazzi, L. Manetti L, M.L. Tanda, E. Dell Unto E, G. Bruno-Bossio, M. Nardi, M.P. Bartolomei, A. Lepri, G. Rosso, E. Martino, A. Pincera, Relation between therapy for hyperthyroidism and the course of Graves'ophthalmopathy. N. Engl. J. Med. 338(2), 73-78 (1998).

18. L. Tallstedt, G. Lundell, O. Torring, G. Wallin, J.G. Ljunggren, H. Blomgren, A. Taube, Occurrence of opthalmopathy after treatment for Graves hyperthyroidism. The thyroid study group. N. Engl. J. Med. 326(26), 1773-1788 (1992).

19. M.A. Gianfrancesco, L. Trupin, S. Shiboski, M. Van der Laan, J. Graf, J. Imboden, J. Yazdany, G. Schmajuk, Smoking is associated with disease activity in rheumatoid arthritis: a longitudinal study controlling for time-varying covariates. J. Rheumatol. 46(4), 370-375 (2019).

20. Y. Tu, G. Fan, Y. Dai Y, T. Zeng, F. Xiao, L. Chen, W. Kong, Association between rs3087243 and rs231775 polymorphism within the cytotoxic T-lymphocyte antigen 4 gene and Graves' disease: a case/control study combined with meta-analyses. Oncotarget 8(66), 110614-110624 (2017).

21. Y. Ban, T.F. Davies, D.A. Greenberg, A. Kissin, B. Marder, B. Murphy et al. Analysis of the CTLA-4, CD28, and inducible costimulator (ICOS) genes in autoimmune thyroid disease. Genes Immunity 4(8), 586-593 (2003). 
22. T. Koukki, Y. Sawai, C.A. Gardine, M. Fisfalen, M. Alegre, L.J. De Groot, CTLA-4 gene polymorphism at position 49 in exon 1 reduces the inhibitory function of CTLA- 4 and contributes to the pathogenesis of Graves' disease. J. Immunol. 165(11), 6606-6611 (2000).

23. D. Saverino, R. Brizzolara, R. Simone, A. Chiappori, F. Militenda-Floriani, G. Pesce, M. Bagnasco, Soluble CTLA-4 in autoimmune thyroid diseases: relationship with clinical status and possible role in the immune response dysregulation. Clin. Immunol. 123(2), 190-198 (2007).
24. J. Daroszewski, E. Pawlak, I. Karabon, I. Frydecka, A. Jonkisz, M. Slowik, M. Bolanowski, Soluble CTLA-4 receptor an immunological marker of Graves' disease and severity of ophthalmopathy is associated with CTLA-4 Jo31 and CT60 gene polymorphisms. Eur. J. Endocrinol. 161(5), 787-793 (2009).

25. D. Saverino, R. Simone, M. Bagnasco, G. Pesce, The soluble CTLA-4 receptor and its role in autoimmune diseases: an update. Auto Immun Highlight 1(2), 73-81 (2010). 